\title{
CARTA DA VEGETAÇÃO NATURAL POTENCIAL DE CALDAS DA RAINHA*
}

\author{
Carlos Neto ${ }^{1}$ \\ Estevẽo PereirA ${ }^{2}$ \\ EusÉBIO REIS ${ }^{3}$ \\ José CARLOS COSTA ${ }^{4}$ \\ JORGE CAPELO 5 \\ CRistina HenRiQues ${ }^{6}$
}

\begin{abstract}
Resumo - A elaboração da Carta de Vegetação Natural Potencial (VNP) de Caldas da Rainha baseou-se no paradigma segundo o qual existe uma correspondência entre a VNP e uma determinada combinação de bioclima, litologia e solos, num dado contexto biogeográfico. Com base nesta combinação é possível estabelecer a relação entre uma dada combinação das variáveis referidas e uma única sequência sucessional (série de vegetação). Com base no modelo já existente sobre as séries de vegetação de Portugal e sua relação com os solos, litologia, bioclima e biogeografia foi possível estabelecer uma matriz de correspondências entre estas variáveis e a VNP na área do concelho de Caldas da Rainha. A carta da VNP daí resultante foi validada com base nas comunidades efectivamente presentes no território e verificou-se que a aplicação do modelo apresentou uma eficácia quase total na determinação da VNP.

Os resultados apresentados neste trabalho indicam que quase toda a área do concelho é ocupada por sobreirais, cercais, olmais e salgueirais potenciais. Os primeiros estão associados a solos podzólicos, ácidos, de areias e arenitos; os cercais ocorrem nos solos derivados de calcários, margas ou dolomias; os salgueirais e olmais estão associados às planícies aluviais das ribeiras do Oeste. Por último, os valores mais importantes para protecção e conservação (pela sua raridade e presença de vários endemismos) estão nos complexos halofílico (Lagoa de Óbidos), psamofílico, rupícola e turfoso.
\end{abstract} em SIG.

Palavras-chave: Séries de vegetação, geomorfologia, litologia, solos, modelação

* Recebido: 26/05/08. Revisto: 1/10/08. Aceite: 23/10/08.

Professor do Departamento de Geografia da FLUL. E-mail: carlosneto@fl.ul.pt

Bolseiro de Doutoramento da FCT. Centro de Estudos Geog. E-mail: estevaohnh@yahoo.com.br Professor do Departamento de Geografia da FLUL. E-mail: eusebioreis@campus.ul.pt

4 Departamento de Protecção de Plantas e de Fitoecologia, I.S.A. E-mail: jccosta@isa.utl.pt

5 Investigador Auxiliar do Inst. Nac. de Recursos Biológicos. E-mail: jorge.capelo@gmail.com

6 Investigadora do Centro de Estudos Geográficos. E-mail: cristina.s.henriques@gmail.com 


\begin{abstract}
A potential natural vegetation map of Caldas da Rainha. This article presents and discusses a Potential Natural Vegetation (PNV) Map of the Caldas da Rainha Municipality. After outlining the concept, an assessment is made of the conditioning factors that affect the PNV (soil, geology, lithology, bioclimate). A model allowing for the estimation of the PNV is then put forward based on a correspondence drawn between the PNV and the relevant biotopic variables. The resulting PNV map was validated through comparison with the vegetal communities effectively present in the field.

The PNV Map of Caldas da Rainha shows that almost the entire area of this Municipality corresponds to potential cork-oak, Portuguese-oak, elm and willow forests. Cork-oak forests are associated with podzolic soils, made up of sand and sandstone; Portuguese-oak forests (Quercus faginea subsp. broteroi) occur in the presence of limestones, clays, and dolomites; whereas willow and elm forests are associated with the floodplains of the hydrographical unit called the Ribeiras do Oeste. Finally, it is worth noting that the most relevant features in terms of protection and conservation (due both to their rarity and to the presence of endemic species) are to be found in complex communities: halophytic (Óbidos lagoon), psammophilous, rupicolous and peat bogs.
\end{abstract}

Key words: Vegetations series, geomorphology, lithology, soils, GIS modelling.

Résumé - Carte de la vegetation naturelle potentielle de Caldas dA RaInHA. L'élaboration d'une Carte de la Végétation Naturelle Potentielle (VNP) de Caldas da Rainha est basée sur la correspondance existante entre la VNP et la combinaison bioclimatique, lithologique et édaphique du contexte biogéographique. Cela permet d'établir la correspondance existant entre une combinaison donnée de ces éléments et la séquence (série) de végétation correspondante. En utilisant les modèles déjà existants pour le Portugal, on a établi une matrice de correspondance valable pour la municipalité de Caldas da Rainha. La carte VNP résultante a été validée en considérant les communautés effectivement présentes et on a constaté l'efficacité presque totale du modèle appliqué. Presque toute l'aire de la municipalité est occupée pas des formations potentielles de chênes-liège, de chênes rouvres, d'ormes et de saules. Les premiers sont associés à des sols podzoliques acides, sur sables et grès, les chênes rouvres poussent sur sols calcaires, marneux ou dolomitiques, les saules et les ormes correspondent aux plaines alluviales. Les espèces les plus dignes de protection et de conservation, en raison de leur rareté et de la présence de diverses endémismes, se trouvent dans les complexes halophiles (Lagune de Óbidos), psammofiles, ripicoles et tourbeux.

Mots-clés: Séries de végétation, géomorphologie, lithologie, sols, modèles SIG.

\title{
I. INTRODUÇÃO
}

\section{Cartografia preditiva e Vegetação Natural Potencial (VNP)}

O conceito de Vegetação Natural Potencial foi introduzido por Tüxen (1956) e foi retomado, amplamente discutido e modificado posteriormente por diversos autores (Tüxen, 1973, 1977, 1978, 1979; Westhoff e Van der Maarel, 1973; Rivas-Martinez, 1976, 1987, 1994, 2005; Kalkhoven e van der Werf, 1988; 
Theurillat, 1992a, 1992b; Moravec, 1998; Rivas-Martínez et al., 1999). Segundo Westhoff e Van der Maarel (1973), a VNP de um território materializa o estádio de máximo desenvolvimento da vegetação num dado habitat onde todas as influências antrópicas cessaram, como também sucede nos territórios contíguos. Desta forma a VNP define-se através de comunidades vegetais que atingiram o seu máximo ecológico estável. Estas comunidades reflectem, na sua organização interna, a resposta mais eficaz às condições do biótopo, responsáveis pelo fornecimento de matéria e energia ao sistema. Desde que estas condições permaneçam inalteráveis a comunidade mantém-se, na sua estrutura, na composição e no seu funcionamento interno e, portanto, a sua permanência como VNP persiste até que essas condições se alterem. Esta visão determinística do processo de sucessão tem sido colocada em causa por estudos recentes que defendem um modelo de dinâmica sucessional como uma cadeia de Markov discreta no tempo, perturbada por "white noise" (Hastings et al., 1993; Stone e Ezrati, 1996; Anand e Orlóci, 1997; Ricotta et al., 2002). Os referidos autores defendem que o processo sucessional apresenta uma primeira fase de determinismo linear mas, a longo prazo, a sua evolução encerra um elevado grau de incerteza.

Apesar do processo sucessional poder assumir um carácter relativamente individual e não exactamente repetível (Carlo et al., 2002), no curto prazo podemos assumir a existência de um carácter determinístico na relação entre uma série de vegetação e um conjunto de variáveis ambientais (Lawrence e Chase, 2007). Com base neste princípio podemos afirmar que, partindo de qualquer ponto de uma série de vegetação, e admitindo que o factor ou factores antrópicos perturbadores desaparecem, a trajectória de sucessão converge para uma comunidade vegetal que corresponde ao máximo ecológico estável (clímax). Assumindo que a sucessão é relativamente determinística, apesar dos aspectos anteriormente referidos, podemos então prever qual a vegetação de máximo ecológico estável (clímax) num determinado ponto de um território, partindo da comunidade vegetal que efectivamente está presente no momento da análise. Por isso, muitos autores chamam "cartografia preditiva" à metodologia subjacente à realização de uma carta da VNP (Miller, 2002; Kelly et al., 2005, Miller et al., 2007). No entanto, um modelo deste tipo, que permite prever com carácter relativamente determinístico a VNP, só poderá estabelecer-se desde que a vegetação de um dado território se encontre devidamente estudada e as séries de vegetação sejam conhecidas. Portanto, a elaboração da Carta da Vegetação Potencial tem de partir da interpretação mais ou menos subjectiva das relações entre os factores ambientais, a vegetação actual e a VNP, realizada por um especialista. A componente subjectiva da análise não lhe retira o seu rigor, pois tem de basear-se num conjunto de trabalhos de investigação prévios sobre a vegetação do território (Ricotta et al., 2000), no qual se irá aplicar o conceito da VNP. O modelo preditivo por si é insuficiente sendo necessário testá-lo com base nas comunidades vegetais efectivamente presentes no território, (Austin, 2007). 
Neste momento, após algumas décadas de intenso trabalho, o conhecimento sobre a vegetação de Portugal encontra-se num nível suficientemente detalhado nas diferentes porções do território, possibilitando a definição teórica das relações entre os principais factores ambientais (climáticos, geomorfológicos e edáficos) e as diferentes séries de vegetação. Assim, foi realizada a primeira Carta da VNP de Portugal Continental na escala 1:1000.000 (Capelo et al., 2007) com recurso a cartografia automática baseada nos modelos já existentes de relação entre os factores ambientais e a VNP, e manualmente corrigida com base no conhecimento dos principais especialistas nacionais. Embora sendo este o primeiro mapa da VNP de Portugal, foram realizadas nas últimas duas décadas diversas cartas para áreas mais ou menos circunscritas, as quais correspondem às primeiras aplicações do conceito da VNP à produção cartográfica de carácter preditivo, em Portugal: Carta das Séries de Vegetação do Sul de Portugal (RivasMartínez et al., 1990); Carta de Séries de Vegetação da Serra da Arrábida (Almeida e Capelo, 1996); Esboço da Paisagem Vegetal da Bacia Portuguesa do Rio Guadiana (Capelo, 1996); Cartografia Fitossociológica da Vegetação do Estuário do Sado e Costa da Galé (Neto et al. 2001); Mapa das Séries de Vegetação do Norte de Portugal (Aguiar, 2001); Carta de VNP das Terras de Sicó (Lopes, 2001); Séries de Vegetação e Subséries Climatófilas do P.N. da PenedaGêres (Honrado, 2003); Mapa das Séries de Vegetação do Ribatejo (Gaspar, 2003); Carta das Séries de Vegetação da Ilha da Madeira (Capelo et al., 2004); Carta da VNP do Barrocal Algarvio (Pinto-Gomes e Ferreira, 2005) e Carta da VNP do Minho e Douro Litoral, Trás-os-Montes e Alto Douro (Almeida e Capelo, 2007).

Na Península Ibérica, o Mapa das Séries de Espanha (Rivas-Martínez, 1975) representa o primeiro trabalho de aplicação cartográfica do conceito de série de vegetação e de VNP.

O conceito de VNP e a sua expressão cartográfica a um território assume uma enorme relevância no planeamento e ordenamento em quase todos os países da Europa (Kalkhovenv e Van der Werf, 1988, Chytrý, 1998; Blasi et al., 2000). A sua aplicação na reconstituição da vegetação natural após acção antrópica tem grande importância, dado o carácter preditivo que o conceito encerra (Zampieri, 2008). Por esta razão, os trabalhos de aplicação prática do conceito de VNP à realização de cartas de Vegetação Natural Potencial têm-se difundido por quase todo o mundo (Küchler, 1964; Burgess, 1965; Kalkhoven e van der Werf, 1988; Brzeziecki et al., 1993; Bohn, 1996; Cross, 1998; Birch, 2000; Bohn et al., 2000; Vuerich et al., 2001; Bohn et al., 2003; Zhao e Feng, 2004; Cross, 2006).

\section{A área de estudo (concelho de Caldas da Rainha)}

Do ponto de vista biogeográfico o concelho de Caldas da Rainha encontra-se situado na Região biogeográfica Mediterrânica, Província Lusitano-Andaluza Litoral, Subprovíncia Divisório Portuguesa-Sadense, Sector Divisório-Português, 
Subsector Divisório-Português Setentrional, e Distrito Estremenho Litoral. O território correspondente a esta última unidade biogeográfica desenvolve-se próximo do litoral, e serviu de via migratória para os elementos florísticos de carácter atlântico no seu deslocamento para Sul durante os períodos mais frescos e húmidos do Quaternário (Costa et al., 1998). Este facto, associado à grande diversidade litológica, à influência do mar e à proximidade da fronteira entre as regiões biogeográficas Mediterrânea e Eurossiberiana, justifica a elevada riqueza da flora e da vegetação. A proximidade da referida fronteira biogeográfica permite a convivência, neste espaço, entre elementos florísticos atlânticos e elementos florísticos de carácter mediterrâneo. Esta convivência justifica a originalidade do elenco florístico de algumas comunidades vegetais que caracterizam o concelho de Caldas da Rainha.

\section{METODOLOGIA}

Do ponto de vista metodológico a realização da Carta da VNP do concelho de Caldas da Rainha baseou-se na Carta dos Solos de Portugal 1:25 000 (IDRHa, 1994) e na Carta Geológica de Portugal 1:50 000, folhas 26-B Alcobaça (Camarate França e Zbyszewski, 1963), 26-D Caldas da Rainha (Zbyszewski e Moitinho de Almeida, 1960) e Folha 30-B Bombarral (Zbyszewski e Veiga Ferreira, 1966). Com base nestas cartas foi criado um modelo de correspondência entre a litologia, a geologia, os solos e a VNP, com uma correcção para as áreas litorais submetidas à influência dos ventos fortes e da salsugem (quadros I e II). O modelo de correspondências desenvolvido teve em conta o facto de todo o concelho de Caldas da Rainha pertencer a um só território biogeográfico e apresentar apenas um bioclima (termomediterrâneo superior) (Mesquita, 2005). Na elaboração do modelo de relações apresentado nos quadros I e II, recorreu-se aos trabalhos já publicados sobre a vegetação do território analisado, assim como ao modelo sobre o qual a equipa tem trabalhado, relativo às séries de vegetação de Portugal e à VNP na sua relação com as variáveis ambientais discriminantes (Capelo et al., 2007). Para além da produção automática da carta da VNP que as relações identificadas nos quadros I e II permitem, o trabalho de campo é essencial na definição da extensão e tipo de influência da proximidade do litoral, assim como na validação do modelo de relações proposto, através da vegetação efectivamente presente no terreno. 


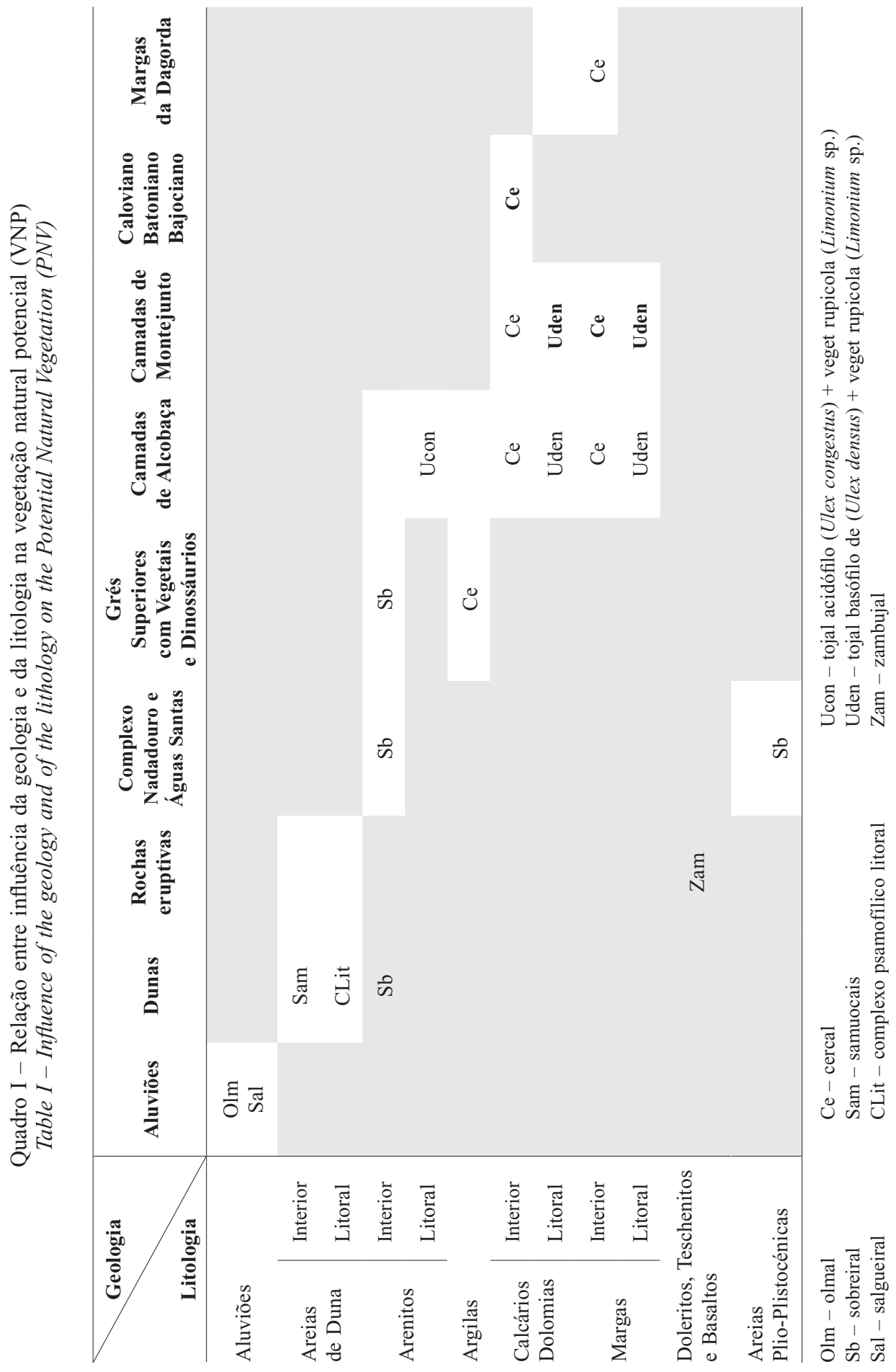


Quadro II - Unidades pedológicas e respectiva vegetação natural potencial Table II - Pedologic units and the respective potential natural vegetation

\begin{tabular}{|c|c|c|c|}
\hline Solo & VNP & Solo & VNP \\
\hline A & Salgueiral & Pcs & Cercal \\
\hline Aac & Salgueiral & Pcs & Cercal \\
\hline Ac & Salgueiral & Pcst & Cercal \\
\hline $\mathrm{Al}(\mathrm{h})$ & Salgueiral & Pdg & Sobreiral \\
\hline Alc & Salgueiral & Ppr & Sobreiral \\
\hline Ap & Sobreiral & $\operatorname{Ppt}(\mathrm{p})$ & Sobreiral \\
\hline Ap (Litoral) & Complexo psamofílico & Ptco & Cercal \\
\hline Aph & Olmal & Pto & Sobreiral \\
\hline Apr & Sobreiral & $\mathrm{Pvb}$ & Zambujal \\
\hline Arc (litoral) & Complexo rupícola acidofilo & $\operatorname{Pvd}(p)$ & Cercal \\
\hline Arc & Cercal & $\mathrm{Pz}$ & Sobreiral \\
\hline Arc (litoral) & Complexo rupícola basófilo & Rcg (Litoral) & Complexo psamofílico \\
\hline Arct (litoral) & Complexo rupícola basófilo & $\mathrm{Sb}$ & Olmal \\
\hline Art (litoral) & Complexo rupícola acidofilo & $\mathrm{Sbc}$ & Olmal \\
\hline As & Complexo halofílico & Sbl & Olmal \\
\hline At & Salgueiral & Sblc & Olmal \\
\hline Atc & Salgueiral & $\mathrm{Sp}$ & Complexo turfoso \\
\hline Atl & Salgueiral & Srt & Sobreiral \\
\hline Bp & Zambujal & Svc & Cercal \\
\hline Bva & Zambujal & Svc (Litoral) & Complexo rupícola basófilo \\
\hline $\mathrm{Ca}$ & Olmal & Vac & Cercal \\
\hline $\mathrm{Cac}$ & Olmal & Vag & Sobreiral \\
\hline $\mathrm{Cal}$ & Olmal & Vagc & Cercal \\
\hline Calc & Olmal & Vago & Sobreiral \\
\hline $\mathrm{Cb}$ & Zambujal & $\operatorname{Val}(\mathrm{h})$ & Olmal \\
\hline $\mathrm{Lb}$ & Zambujal & Vatc & Cercal \\
\hline $\operatorname{Lbd}(p)$ & Zambujal & Vato & Sobreiral \\
\hline $\operatorname{Lpt}(\mathrm{a})$ & Sobreiral & Vato (Litoral) & Complexo rupícola acidófilo \\
\hline Paco & Cercal & $\mathrm{Vc}$ & Cercal \\
\hline Paco & Cercal & Vcd (Litoral) & Complexo rupícola basófilo \\
\hline Pag & Sobreiral & $\operatorname{Vcd}(\mathrm{a})$ & Cercal \\
\hline Pagc & Cercal & Vcmo & Cercal \\
\hline Pago & Sobreiral & Vest & Cercal \\
\hline Par & Sobreiral & Vdg & Sobreiral \\
\hline Pato & Sobreiral & $\mathrm{V} 1$ & Sobreiral \\
\hline $\mathrm{Pbd}$ & Zambujal & $\mathrm{Vt}$ & Sobreiral \\
\hline $\mathrm{Pc}$ & Cercal & Vt (Litoral) & Complexo rupícola acidófilo \\
\hline Pcdc & Cercal & $\mathrm{Vt}$ & Sobreiral \\
\hline
\end{tabular}

Os códigos dos solos seguem a Classificação usada na Carta dos Solos de Portugal 1:25000 IDRHa (1994) 


\section{DESCRIÇÃO DAS UNIDADES DE VEGETAÇÃO POTENCIAL}

\section{As formações vegetais florestais potenciais}

\subsection{Cercais}

Quanto às florestas potenciais, o concelho de Caldas da Rainha é dominado por sobreirais e cercais. Os bosques de carvalho-cerquinho (Quercus faginea subsp. broteroi) constituem as florestas dominantes (fig. 1), devido ao predomínio dos solos básicos derivados de margas e calcários. Trata-se de bosques fechados com mais de 10 metros de altura, onde o carvalho-cerquinho ocorre com uma taxa de cobertura frequentemente superior a 50\%. Acompanhando o carvalho-cerquinho no estrato arbóreo é frequente a ocorrência de loureiros (Laurus nobilis), adernos (Phillyrea latifolia), medronheiros (Arbutus unedo), zambujeiros (Olea sylvestris) e raros sobreiros (Quercus suber). No estrato arbustivo são frequentes: folhado (Viburnum tinus), aroeira (Pistacia lentiscus), sanguinho-das-sebes (Rhamnus alaternus), urze-branca (Erica arborea), murta (Myrtus communis), gilbardeira (Ruscus aculeatus) e Euphorbia characias. Junto ao solo desenvolve-se um estrato herbáceo dominado por plantas vivazes (Asplenium onopteris, Luzula forsteri, Arisarum vulgare, Carex distachya, Iris foetidissima, Arum italicum). O estrato de trepadeiras está, também, bem representado: Hedera hibernica, Rubia longifolia, Rosa sempervirens, Lonicera hispanica, Tamus communis, Rubus ulmifolius.

Os cercais são frequentes no Maciço Calcário Estremenho, na Serra da Arrábida e ainda nos calcários jurássicos e cretácicos situados a norte de Lisboa, sempre em ambientes mesófilos. Embora não sendo um tipo de bosque raro do ponto de vista potencial, os exemplares em bom estado de conservação são raros, pelo que deram origem a sítios da Rede Natura 2000. No Anexo II da Directiva Habitats, as florestas de Quercus broteroi constituem um habitat protegido, não prioritário (9240 - "Florestas ibéricas de Quercus faginea e Quercus canariensis", ALFA, 2004). Das etapas regressivas dos cercais, a que apresenta maior índice de originalidade, é o loureiral. É uma formação subarbórea, mais raramente arbustiva, dominada por loureiros ( $L a u$ rus nobilis) que ocupam solos derivados de calcários com compensação edáfica. Não suportam seca extrema e portanto são dominantes nas vertentes sombrias, áreas deprimidas ou vales fechados. Nestas situações topográficas os loureirais ocorrem como primeira etapa de substituição ou orla mais húmida dos cercais.

Os loureirais do concelho de Caldas da Rainha constituem uma comunidade vegetal endémica de Portugal, que aqui apresenta a sua maior área de distribuição. Do ponto de vista da protecção e conservação apresentam um interesse elevado. Estão integrados na Rede Natura $2000(* 5230$ - Matos arborescentes de Laurus nobilis, ALFA, 2004), onde são considerados prioritários devido ao seu carácter reliquial e pelo facto de integrarem, de modo geral, numerosos elementos florísticos paleo-subtropicais (terciários) de características lauróides, 


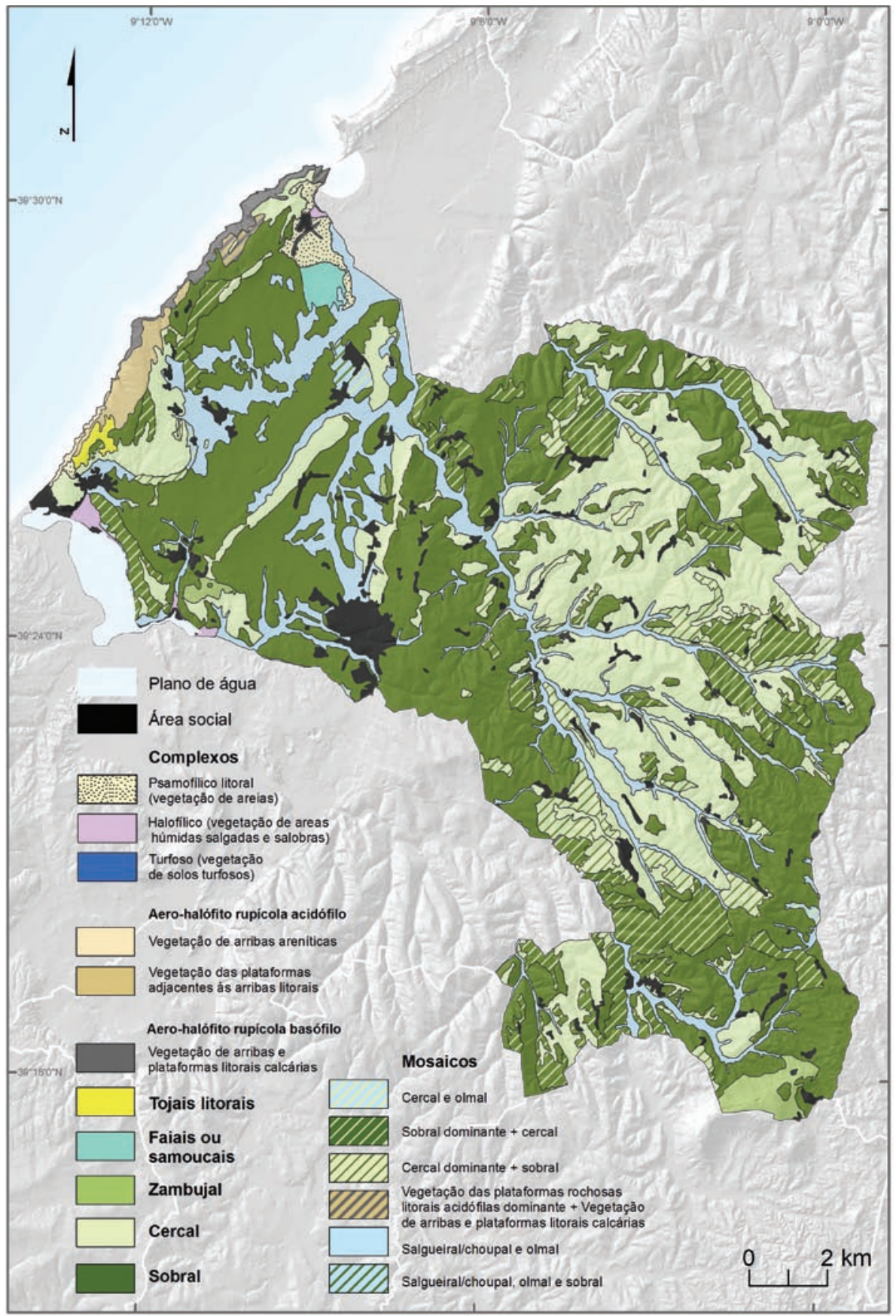

Sistema de Projecção Hayford - Gauss Datum 73

Fig. 1 - Carta da Vegetação Natural Potencial do concelho de Caldas da Rainha Fig. 1 - Potential Natural Vegetation map of Caldas da Rainha 
como Prunus lusitanica, Phillyrea latifolia, Arbutus unedo, Laurus nobilis e Myrica faya.

Do ponto de vista das formações litológicas e geológicas, os cercais estão potencialmente associados aos solos derivados de calcários, margas, argilas e dolomias (Margas de Dagorda, Camadas de Montejunto, Camadas de Alcobaça, Grés Superiores com Vegetais e Dinossáurios, Batoniano e Bajociano e Calcários e Dolomias de Dagorda).

\subsection{Sobreirais}

Os sobreirais correspondem à segunda formação vegetal potencial, quanto à dominância no concelho de Caldas da Rainha (fig. 1). Este facto deve-se à presença de uma extensa cobertura de areias, arenitos e cascalheiras de idade plio-plistocénica (principalmente do Complexo Astiano de Nadadouro e Águas Santas), sobre as quais se originaram solos acidófilos de natureza arenosa. Ao contrário do que sucede com o carvalho-cerquinho, que prefere solos derivados de calcários, o sobreiro é uma espécie calcífuga e acidófila. Desta forma encontramos os sobreirais potenciais associados à grande extensão de solos derivados de materiais arenosos, com pH baixo (solos ácidos), maioritariamente podzolizados. Apesar de potencialmente ocuparem uma porção significativa do território do concelho de Caldas da Rainha, a presença de sobreirais em bom estado de conservação é pouco frequente.

São formações florestais frequentemente com mais de 10 metros de altura e enorme densidade, dominadas no estrato arbóreo pelo sobreiro (Quercus suber), medronheiro (Arbutus unedo), aderno (Phillyrea latifolia), zambujeiro (Olea europaea var. sylvestris), carvalho-cerquinho (Quercus faginea subsp. broteroi), carvalho-negral (Quercus pyrenaica) e castanheiro (Castanea sativa). O estrato arbustivo é dominado por espécies como Asparagus aphyllus, Daphne gnidium, Rhamnus alaternus, Quercus coccifera, Pistacia lentiscus, Myrtus communis, Osyris alba, Ruscus aculeatus, Quercus lusitanica, Viburnum tinus e Erica arborea. O estrato de trepadeiras é constituído por Smilax aspera, Rubia longifolia, Hedera hibernica, Rosa sempervirens, Lonicera estrusca, Lonicera implexa, Rubus ulmifolius, Tamus communis e Lonicera hispanica. No estrato herbáceo ocorre Arisarum vulgare, Vinca difformis, Asplenium onopteris, Deschampsia stricta, Carex distachya, Scilla monophyllos e Luzula baetica (Costa et al., 2002).

Quanto à corologia, os sobreirais silicícolas que ocorrem no concelho de Caldas da Rainha correspondem a uma comunidade vegetal relativamente frequente no Centro e no Sul de Portugal, particularmente nos solos derivados das areias, arenitos e conglomerados do Pliocénico e Plistocénico das bacias do Tejo e do Sado.

No Anexo II da Directiva Habitats os sobreirais constituem um habitat protegido, embora não prioritário (9330 - Florestas de Quercus suber, ALFA, 2004).

\subsection{Zambujais}

Os zambujais (florestas mediterrâneas de oliveira-brava) correspondem a um tipo florestal pouco frequente em Portugal. Estão sempre associadas à 
ocorrência de solos com propriedades vérticas, devidas à dominância de argilas de forte expansibilidade. A dominância deste tipo de argilas ocorre em alguns solos derivados de calcários; no entanto, é nos afloramentos de materiais vulcânicos (Complexo Vulcânico de Lisboa) que os solos com propriedades vérticas são dominantes. Desta forma, as florestas de zambujeiro com alfarrobeiras apresentam distribuição Arrabidense e Olissiponense. No concelho de Caldas da Rainha os zambujais apresentam a distribuição mais setentrional em Portugal; no entanto a sua ocorrência neste concelho é relativamente pouco vulgar (fig. 1) e sucede apenas em associação com os solos derivados de doleritos.

Os zambujais constituem um habitat protegido do Anexo II da Directiva Habitats (9320 - Florestas de Olea e Ceratonia, ALFA, 2004), embora só os locais em que esta comunidade se encontra presente em bom estado de conservação tenham sido considerados como sítios da Rede Natura 2000, o que não sucede no concelho de Caldas da Rainha. Estruturalmente constituem florestas de zambujeiros, por vezes com mais de 10 metros de altura, de copado denso e com sub-bosque arbustivo latifoliado/espinhoso, lianóide e herbáceo vivaz, desenvolvido. O estrato arbóreo é formado por zambujeiros (Olea europaea var. sylvestris) de forma quase pura, acompanhados por adernos (Phillyrea latifolia), freixos (Fraxinus angustifolia), olmeiros (Ulmus minor) e loureiros (Laurus nobilis), nos locais com alguma compensação edáfica. No estrato arbustivo aparece um grande número de arbustos perenifólios de características mediterrânicas: Rhamnus alaternus, Viburnum tinus, Ruscus aculeatus, Asparagus aphyllus, Pistacia lentiscus, Quercus coccifera, Daphne gnidium, Euphorbia characias, Osyris alba, Prunus insititioides, Jasminum fruticans, Paeonia broteroi e Erica arborea. No estrato aéreo ocorrem numerosas trepadeiras: Smilax aspera, Rubia longifolia, Bryonia dioica, Rosa sempervirens, Lonicera implexa, Lonicera etrusca, Hedera hibernica, Rubus ulmifolius e Lonicera hispanica. Quanto ao estrato herbáceo, é composto por Arum italicum subsp.neglectum, Arum italicum, Vinca difformis, Arisarum vulgare, Acanthus mollis, Carex distachya, Iris foetidissima e Hyacinthoides hispanica.

\subsection{Samoucais/Medronhais}

Os medronhais com samouco (ou faia-das-ilhas) ocorrem como bosques potenciais nas dunas antigas estabilizadas e afastadas da influência do mar. Trata-se de um microbosque dominado por espécies latifoliadas (de folha grande): medronheiro (Arbustus unedo), samouco (Myrica faya), folhado (Viburnum tinus) e loureiro (Laurus nobilis) (Capelo e Mesquita, 1999). No estrato arbustivo, para além do folhado ocorrem Erica arborea, Phillyrea angustifolia, Cytisus cabezudoi, Rhamnus alaternus, Daphne gnidium, Corema album, Pistacia lentiscus, Ruscus aculeatus e Quercus coccifera. No estrato aéreo de trepadeiras é frequente a Rubia longifolia e Smilax aspera. O estrato herbáceo está fracamente representado.

Os bosques de samouco e medronheiros correspondem à etapa clímax (mais evoluída) da série de vegetação que ocorre nas dunas interiores, afastadas da 
influência da salsugem e frequentemente podzolizadas, entre o Cabo da Roca e Aveiro. Do ponto vista da Rede Natura 2000 foram considerados como um habitat prioritário (5230*, subtipo 5230 pt4 - "Matagais arborescentes de Laurus nobilis", ALFA, 2004).

\subsection{Choupais/Salgueirais de salgueiro-branco}

Os choupais/salgueirais de salgueiro-branco (Salix neotricha) correspondem aos bosques ripícolas potenciais do concelho de Caldas da Rainha. Ocorrem ao longo dos rios e ribeiras do concelho, ocupando as margens ou as planícies aluviais, pelo menos de forma potencial. As "Ribeiras do Oeste" têm como característica comum apresentarem uma elevada riqueza em nutrientes (águas eutróficas), motivada pela natureza calcária do substrato do sector mais a montante e, também, pela agricultura e produção animal intensivas que caracterizam estas bacias hidrográficas. A água, que flui nos referidos cursos de água, apresenta sempre uma grande riqueza em cálcio, em nutrientes (fósforo, azoto, nitritos e nitratos), uma grande quantidade de sólidos suspensos e, ainda, valores elevados de $\mathrm{pH}$ e dureza. Devido à forte acção antrópica os salgueirais/choupais apresentam uma área de distribuição exígua em todo o concelho, o que contrasta com a sua área de distribuição potencial extensa, maioritariamente ocupada por terrenos de cultivo.

Do ponto de vista florístico, os salgueirais/choupais do concelho de Caldas da Rainha são dominados no estrato arbóreo pelo salgueiro-branco (Salix neotricha) e choupo-negro (Populus nigra) acompanhados por outras árvores como o freixo (Fraxinus angustifolia), borrazeira-negra (Salix atrocinerea), amieiro (Alnus glutinosa) e ulmeiro (Ulmus minor). Apresentam sempre um sub-bosque arbustivo e subarbustivo de Frangula alnus, Sambucus nigra, Tamarix africana, Salix salvifolia, Crataegus monogyna subsp. brevispina, Laurus nobilis e diversas plantas nemorais de que são exemplo Arum italicum subsp. neglectum, Vinca difformis, Equisetum telmateia, Carex pendula, Iris foetidissima, Solanum dulcamara, Brachypodium sylvaticum, Scrophularia scorodonia, Ruscus aculeatus, Silene latifolia e Saponaria officinalis. Apresentam ainda um importante estrato de lianas como Clematis campaniflora, Humulus lupulus, Hedera hibernica, Rosa sempervirens, Tamus communis, Rubus ulmifolius, Lonicera periclymenum subsp. hispanica, Calystegia sepium, Vitis vinifera.

Os salgueirais/choupais presentes no concelho de Caldas da Rainha estão referenciados no Anexo II da Directiva Habitats da Rede Natura 2000 como um habitat protegido, não prioritário (92AO, subtipo 92AOpt2 - Florestas-galerias de Salix alba e Populus alba ALFA, 2004).

\subsection{Olmais}

Nas áreas mais afastadas dos cursos de água, mas ainda na planície aluvial, com toalha freática próxima da superfície, ocorre um bosque dominado por olmeiro (Ulmus minor). Esta comunidade é a que ocupa maior área dentro das planícies aluviais dos rios e ribeiras do concelho de Caldas da Rainha. Contudo, 
a sua presença é escassa devido à grafiose que tem dizimado grande quantidade de olmeiros em toda a Europa e principalmente devido à substituição dos olmais por campos agrícolas. O olmal é uma formação arbórea dominada por ulmeiros (Ulmus minor), acompanhados por choupo-preto (Populus nigra), freixo (Fraxinus angustifolia), salgueiro-branco (Salix neotricha), amieiro (Alnus glutinosa) e borrazeira-negra (Salix atrocinerea). No estrato arbustivo e subarbustivo são frequentes: sabugueiro (Sambucus nigrae), pilriteiro (Crataegus brevispina), sanguinho-de-água (Frangula alnus) e Scrophularia scorodonia. No estrato herbáceo são frequentes: Arum italicum subsp. neglectum, Vinca difformis, Equisetum telmateia, Iris foetidissima, Carex pendula, Silene latifolia e Brachypodium sylvaticum. No estrato aéreo de trepadeiras ocorre: Tamus communis, Hedera hibernica, Rosa sempervirens, Humulus lupulus, Bryonia cretica subsp. dioica e Smilax aspera.

Do ponto de vista da Rede Natura 2000, os olmais que ocorrem no concelho de Caldas da Rainha constituem um habitat protegido, referenciado no Anexo II da Directiva (91F0 - Florestas mistas de Quercus robur, Ulmus laevis, Ulmus minor, Fraxinus excelsior ou Fraxinus angustifolia das margens dos grandes rios (Ulmenion minoris), ALFA, 2004).

\section{Formações vegetais arbustivas e herbáceas potenciais}

\subsection{Complexo Psamofilico Litoral (Praias e dunas litorais recentes)}

A influência do mar, associada, por vezes, à mobilidade do substrato arenoso e à oligotrofia do solo, impossibilita a existência de florestas. Nestas situações as comunidades herbáceas e arbustivas tornam-se as comunidades mais evoluídas do sistema. As comunidades litorais próprias das areias sucedem-se da praia para o interior, acompanhando uma melhoria nas condições ecológicas. Esta sucessão tem como consequência que cada comunidade ocupa uma faixa relativamente exígua, mais ou menos paralela ao mar. Assim, apenas em cartas com grande escala é possível representar o espaço ocupado por cada uma das referidas comunidades e, por isso, na carta da vegetação potencial este conjunto está representado apenas por um complexo de comunidades vegetais psamofílicas. Este complexo é constituído por comunidades de elevado interesse para protecção e conservação e por isso todas elas definem habitats protegidos pela Rede Natura 2000. A unidade de vegetação cartografada como complexo psamofílico litoral é constituída pelas comunidades vegetais que a seguir se descrevem.

a) Vegetação anual das zonas de acumulação de detritos pela maré. Corresponde ao habitat 1210 do Anexo II da Directiva Habitats (ALFA, 2004). No concelho de Caldas da Rainha este habitat está representado pela comunidade anual dominada por Cakile maritima, acompanhada por Salsola kali, Beta maritima, e Euphorbia peplis. Trata-se de uma comunidade psamófila, pobre em espécies, constituída por terófitos, 
instalados imediatamente acima da faixa inter-marés, sobre os detritos orgânicos trazidos pelo mar.

b) Dunas móveis embrionárias. Corresponde ao habitat 2110 do Anexo II da Directiva Habitats (ALFA, 2004). No concelho de Caldas da Rainha este habitat está representado pela comunidade dominada pela junça-das-areias (Elytrigia juncea, representado pelas subsp. juncea e subsp. boreoatlantica). Esta comunidade vivaz, pobre em espécies, ocupa a praia alta e sectores mais elevados da praia média afectados pelas vagas durante as tempestades e marés vivas e, por isso, sujeitos a forte movimentação das areias. A junça-das-areias forma, frequentemente, comunidades quase puras, sendo no entanto acompanhada muitas vezes por Eryngium maritimum, Euphorbia paralias, Calystegia soldanella e Pancratium maritimum.

c) Dunas móveis do cordão dunar com Ammophila arenaria ("dunas brancas"). Corresponde ao habitat 2120 do Anexo II da Directiva Habitats (ALFA, 2004). No concelho de Caldas da Rainha este habitat está representado pela comunidade de estorno (Ammophila australis). As dunas móveis são também designadas por dunas vivas, instáveis ou primárias, pois as partículas arenosas não se encontram estabilizadas. Do ponto de vista florístico a duna móvel é dominada por comunidades de Ammophila australis com elevados graus de abundância-dominância e sociabilidade. São frequentes as seguintes espécies características: Ammophila australis, Othantus maritimus, Eryngium maritimum, Calystegia soldanella, Euphorbia paralias, Pancratium maritimum, Lotus creticus e Medicago marina.

d) Dunas fixas com vegetação herbácea (“dunas cinzentas”). Corresponde ao habitat 2130* do Anexo II da Directiva Habitats. Trata-se de um habitat prioritário pois integra muitas espécies endémicas de elevado valor para a conservação. Está representado no concelho de Caldas da Rainha pelo subtipo "Duna cinzenta com matos camefíticos dominados por Armeria welwitschii (2130pt2)" (ALFA, 2004). Os matos dominados por Armeria welwitschii ocupam as dunas semifixas entre o Rio Tejo e Quiaios. Para o alto valor deste habitat contribui a presença de alguns endemismos ibéricos como Armeria welwitschii, Artemisia crithmifolia, Helichysum picardi e Linaria decumbens.

e) Dunas litorais com zimbros (Juniperus spp.). Corresponde ao habitat prioritário 2250* do Anexo II da Directiva Habitats. Está representado no concelho de Caldas da Rainha pela formação arbustiva densa e alta de zimbro (Juniperus turbinata), a qual corresponde ao subtipo "2250pt1 Dunas e paleodunas com matagais de Juniperus turbinata" (ALFA, 2004). Trata-se de comunidades arbustivas litorais ou sublitorais, altas, xerófílas, dominadas por Juniperus turbinata, correspondendo às comunidades lenhosas maduras das dunas estabilizadas litorais ainda marginalmente submetidas à influência directa da salsugem. 
No território estudado a comunidade é muito rara devido à acção antrópica. Apresenta um estrato arbustivo alto dominado por Juniperus turbinata, acompanhado por Daphne gnidium, Pistacia lentiscus, Phillyrea angustifolia, Rhamnus alaternus, Corema album, Myrtus communis e Osyris alba. O estrato de trepadeiras é constituído por Rubia longifolia, Smilax aspera, Antirrhinum cirrhigerum, Lonicera implexa e Hedera helix. O estrato herbáceo está representado por Scilla monophyllos, Geranium purpureum, Geranim molle, Stellaria media, Urtica dubia e Urtica membranacea (Costa et al., 2002).

\subsection{Vegetação das arribas e plataformas rochosas anexas}

As arribas e plataformas rochosas anexas constituem a unidade litoral de maior extensão. Separam-se em arribas e plataformas rochosas areníticas de natureza ácida, dominantes na metade meridional (grés superiores com vegetais e dinossáurios) e arribas e plataformas rochosas calcárias na metade mais setentrional (Camadas de Alcobaça e Camadas de Montejunto).

\subsubsection{Vegetação das arribas}

A vegetação das arribas é dominada por plantas aero-halófilas que suportam simultaneamente a acção do sal e dos ventos fortes. Desta forma a vegetação que aí se instala é extremamente especializada e, portanto, comporta um elevado grau de endemicidade (principalmente ao nível das espécies do género Limonium sp.) e de elevado interesse para conservação. A separação entre as arribas areníticas acidófilas e as calcárias faz-se pela ocorrência dos tojais acidófilos de Ulex congestus nas primeiras e pela ocorrência dos tojais basófilos de Ulex densus nas segundas. Quanto à vegetação dominada por limónios, as comunidades são aparentemente indiferentes à mudança de substrato.

a) Arribas com vegetação das costas mediterrânicas com Limonium spp. endémicas. Corresponde ao habitat 1240 da Directiva Habitats (ALFA, 2004). Nas arribas do concelho de Caldas da Rainha, sob a influência dos ventos marítimos carregados de sal (salsugem), ocorre uma comunidade perene, de fraco grau de cobertura, constituída por pequenos geófitos, caméfitos e hemicriptófitos rupícolas, entre os quais se conta um elevado número de microendemismos. Esta comunidade, dominada por espécies do género Limonium spp., apresenta grande diversidade florística, provavelmente como consequência das condições ecológicas extremas dos habitats de arriba marítima, da elevada taxa de especiação intrínseca aos géneros Limonium e Armeria e da diversidade litológica. Nas arribas da área estudada, ocorrem quatro importantes endemismos lusitanos: Limonium plurisquamatum, Plantago occidentalis, Limonium ovalifolium e Armeria cinera. Outras espécies frequentes são: Dactylis marina, Crithmum maritimum, Daucus halophilus, Calendula algarbiensis, Spergularia australis, Limonium virgatum e Helichrysum decumbens. 
Devido à quantidade de endemismos presentes e à reduzida área de distribuição, as comunidades rupícolas que caracterizam as arribas do concelho de Caldas da Rainha apresenta um elevado grau de valor para protecção e conservação.

b) Arribas calcárias com matos de Ulex densus. Corresponde ao habitat 5330 (subtipo 5330pt7) "Matos termomediterrânicos pré-desérticos", da Rede Natura 2000 (ALFA, 2004). No concelho de Caldas da Rainha está representado pelos matos baixos calcícolas dominados por um tojo endémico do centro-oeste de Portugal (Ulex densus). Este tojal ocupa pequenas plataformas calcárias que se definem nas arribas (rechãs) e em locais onde a inclinação é menor. Distribui-se pelas arribas e plataformas rochosas anexas, entre o Cabo Espichel e o Cabo Mondego. O facto de estar sujeita a constante acção do vento forte, do sal, da pobreza do solo em nutrientes e da sua falta de espessura, justifica o facto de se assumir como comunidade permanente, ou seja, constitui a comunidade vegetal mais evoluída do sistema. Trata-se de um mato baixo dominado por um importante endemismo calcícola do oeste de Portugal (Ulex densus). Embora protegido pela legislação da Rede Natura 2000, não se trata de um habitat prioritário; contudo a sua diversidade florística é enorme e portanto a sua protecção e conservação é importante.

O tojal de Ulex densus é dominado por caméfitos que raramente registam mais de $50 \mathrm{~cm}$ de altura. Floristicamente é constituído pelas seguintes espécies características: Salvia sclareoides, Eryngium dilatatum, Anthyllis maura, Carex hallerana, Daucus crinitus, Bartsia aspera, Serratula estremadurensis, Phagnalon rupestre e Iberis microcarpa.

c) Arribas areníticas com matos de Ulex congestus. Corresponde ao habitat 4030 (subtipo 4030pt1) (ALFA, 2004). "Tojais e urzais-tojais aero-halófilos mediterrânicos". Nos locais de menor inclinação ou em pequenas rechãs, nas arribas areníticas acidófilas ocorrem os tojais e urzais-tojais aero-halófilos amoitados mediterrânicos dominados por Ulex jussiaei ssp. congestus. São interpretados como comunidades permanentes, pois as condições do biótopo não permitem a sua substituição por comunidades mais evoluídas. Trata-se de um habitat pouco comum, que ocorre apenas no sector Divisório-Português. Do ponto de vista florístico os tojais de Ulex congestus são constituídos pelas seguintes espécies características: Daphne maritima, Dactylis marina, Carlina major, Calluna vulgaris, Pulicara odora, Ulex minor e Simethis mattiazii.

\subsubsection{Plataformas rochosas anexas ao oceano}

Assim como sucede nas arribas, nas plataformas rochosas anexas, a vegetação sofre também a influência quase constante dos ventos marítimos carregados de sal. Os biótopos definidos pelas referidas plataformas rochosas apresentam solos pobres, com frequência peliculares e fissurícolas, que condicionam o tipo de flora e a vegetação que aí ocorre. No concelho de Caldas da 
Rainha estas plataformas desenvolvem-se sobre dois tipos de substratos (já referidos para as arribas): os arenitos e os calcários. No sector correspondente aos arenitos (sector meridional) ocorrem os matos de Ulex congestus; no sector setentrional, correspondente aos afloramentos calcários, ocorrem os matos de Ulex densus. Para além destas duas comunidades vegetais, as plataformas rochosas anexas às arribas são também dominadas, nos locais mais abrigados, por carrascais/zimbrais de Juniperus turbinata e carrasco (Quercus coccifera). Corresponde ao habitat 5210 (subtipo 5210pt2) "Zimbrais-carrascais de Juniperus turbinata sobre calcários" da Directiva Habitats da Rede Natura 2000 (ALFA, 2004). Os carrascais/zimbrais correspondem a uma formação arbustiva (microfanerofítica), densa, dominada por espécies esclerófilas que é frequente nas plataformas litorais, principalmente de natureza calcária, onde as condições dos biótopos não possibilitam o desenvolvimento de florestas. Trata-se de um mato pré-florestal (maquis) onde ocorrem lianas xerofíticas como Asparagus aphyllus, Smilax aspera, Rubia longifolia, Lonicera implexa e Rosa sempervirens. Entre os arbustos característicos mais frequentes ocorrem: Daphne gnidium var. maritima, Rhamnus alaternus, Pistacia lentiscus, Phillyrea angustifolia, Olea europaea var. sylvestris, Osyris alba, Myrtus communis e Ruscus aculeatus. Junto ao solo a falta de luz não possibilita o desenvolvimento de um importante estrato herbáceo; contudo, ocorrem as seguintes espécies: Arisarum vulgare, Melica arrecta, Hyacintoides hispanica, Scilla monophyllos e Carex distachya.

\section{CONCLUSÃO}

A geologia do concelho de Caldas da Rainha é dominada pelos "Grés Superiores com Vegetais e Dinossáurios" do Jurássico Superior, que ocupam 63,8\% da área total do concelho. Litologicamente esta formação é constituída por arenitos e argilas onde predominam os solos derivados de materiais não calcários (Litólicos não Húmicos de Arenitos, Mediterrânicos Pardos e Mediterrânicos Vermelhos ou Amarelos), os quais representam 54\% da área ocupada pelo Jurássico Superior. Associados a estes solos não calcários, verifica-se um domínio da vegetação potencial acidófila (sobreirais do Asparago-Quercetum suberis). Os carvalhais potenciais de Quercus broteroi (Arisaro-Quercetum broteroi) aparecem nos solos derivados de materiais calcários em cerca de 39\% da referida unidade geológica.

Em 17,4\% da área do concelho ocorre o "Complexo Astiano de Nadadouro e Águas Santas", do Plio-Plistocénico, constituído por areias com calhaus rolados e grés argilosos. Em função desta litologia, verifica-se um domínio dos solos podzolizados (64\% da área ocupada pela unidade geológica) e, consequentemente, $74 \%$ da área apresenta como vegetação potencial, sobreirais de Asparago-Quercetum suberis. Esta unidade apresenta, ainda, uma importante cobertura superficial de solos de aluvião com salgueirais de Salix neotricha como vegetação potencial ( $22 \%$ da área ocupada pela unidade geológica). As Margas de Dagorda 
(margas, margas gresosas e alguns calcários ou dolomias) afloram em cerca de $3,3 \%$ da área do concelho e apresentam como vegetação potencial dominante, os carvalhais de Quercus faginea subsp. broteroi (36\% da área ocupada pela unidade geológica) em solos derivados de materiais calcários. Os sobreirais potenciais ocupam $29 \%$ da área, encontrando-se sobre solos podzolizados, devido às frequentes coberturas peliculares de areias transportadas a partir do Complexo Pliocénico. Os salgueirais de $S$. neotricha ocorrem em $24 \%$ da unidade geológica, associados às aluviões dos cursos de água. Ocupando 4,2\% da área total do concelho aparece o Oxfordiano (Camadas de Montejunto e Camadas de Alcobaça) formado por calcários, margas e grés. Nesta unidade geológica dominam os solos derivados de calcários e consequentemente a vegetação potencial dominante são os carvalhais de $Q$. faginea subsp. broteroi. Os afloramentos de doleritos ocupam apenas $0,5 \%$ da área total do concelho e são dominados por solos pouco frequentes na região "Solos derivados de Dioritos, Gabros e outras Rochas Cristalofilicas Básicas" os quais ocupam 69\% desta unidade geológica e que, devido às suas propriedades vérticas têm como vegetação potencial os zambujais do Viburno tini-Oleetum sylvestris.

Embora ocupando um escassa extensão, a vegetação natural potencial que ocorre no litoral apresenta enorme importância pois inclui algumas plantas e comunidades endémicas de Portugal, protegidas pela Legislação Portuguesa e da U.E. Nesta unidade litoral, estão incluídos dois conjuntos de comunidades: psamofílicas (dunares e de praia) e rupícolas aero-halófilas (de arribas).

As principais comunidades vegetais identificadas estão correlacionadas com o grau e tipo de acção antrópica, com o tipo de solo, com a profundidade da toalha freática e com a proximidade do mar. Como se trata de uma área fortemente antropizada, a vegetação actual está quase totalmente determinada pela acção humana. Desta forma, os bosques naturais primitivos (que ocupariam a área antes da acção humana mais intensiva) são muito raros e quando ocorrem encontram-se frequentemente alterados na composição e na estrutura.

\section{BIBLIOGRAFIA}

Aguiar C (2001) Flora e vegetação da Serras da Nogueira e do Parque Natural da Montesinho. Dissertação de doutoramento, Instituto Superior de Agronomia, Lisboa.

ALFA (2004) Fichas dos habitats do Plano Sectorial da Rede Natura 2000. ICN, Lisboa.

Alison K (2005) Predicting potential natural vegetation in an interior northwest landscape using classification tree modeling and a GIS. Western Journal of Applied Forestry, 20(2): 117-127.

Almeida A F, Capelo M (2007) Bioclimatologia e vegetação natural potencial. Silva Lusitana, 15(2): 279-284.

Almeida A F, Capelo J (1996) Carta de séries de vegetação da Serra da Arrábida. In Notas do Herbário da Estação Florestal Nacional (LISFA). Fasc. IV. Silva Lusitana, 4(2): 259-264.

Anand M, Orlóci L (1997) Chaotic dynamics in a multispecies community. Environ. Ecol. Stat., 4: 337-344. 
Austin M (2007) Species distribution models and ecological theory: a critical assessment and some possible new approaches. Ecological Modelling, 200: 1-19.

Birch N V E (2000) The vegetation potencial of natural rangelands in the Midfish River valley, Eastern Cape, South Africa: towards a sustainable and acceptable management system. Doctor thesis, Rhodes University, Grahamstown.

Blasi C, Carranza M L, Frondoni R, Rosati L (2000) Ecosystem classification and mapping: a proposal for italian landscapes. Applied Vegetation Science, 3: 233-242.

Bohn U. (1996) Vegetationskarte der Bundesrepublik Deutschland 1: 200000 - Potentielle natürliche Vegetation - Blatt CC 5518 Fulda, Schriftenreihe für Vegetationskunde 15.

Bohn U, Gollub G, Hettwer C (2000) Map of the natural vegetation of Europe. Federal Agency for Nature Conservation, Bonn-Bad Godesberg.

Bohn U, Gollub G, Hettwer C, Neuhäuslová Z, Schlüter H, Weber H (2003) Map of the natural vegetation of Europe 1-3. Federal Agency for Nature Conservation, Bonn-Bad Godesberg.

Brzeziecki B, Kienast, F, Wildi, O (1993) A simulated mapa of the potential natural forest vegetation of Switzerland. Journal of Vegetation Science, 4: 499-508.

Burgess R L (1965) Potential vegetation of the United States. Ecology, 46(4): 568-570.

Camarate França J, Zbyszewski G (1963) Carta Geológica de Portugal na escala de 1/50 000. Noticia explicativa da folha 26-B Alcobaça. Serviços Geológicos de Portugal, Lisboa.

Capelo J (1996) Esboço da paisagem vegetal da bacia portuguesa do Rio Guadiana. Silva Lusitana, 4 (especial): 13-64.

Capelo J, Almeida A F (1993) Dados sobre a paisagem vegetal do parque natural da Serra da Arrábida: proposta de uma tipologia fitossociológica. Silva Lusitana, 1(2): 217-236.

Capelo J, Mesquita S (1999) Nota sobre a vegetação natural e potencial das dunas estabilizadas do superdistrito costeiro português. Silva Lusitana, 6(2): 257-259.

Capelo J, Mesquita S, Costa J C, Ribeiro S, Arsénio P, Neto C, Monteiro-Henriques T, Aguiar C, Honrado J, Espírito-Santo D, Lousã M (2007) A methodological approach to potential vegetation modeling using gis techniques and phytosociological expert-knowledge: application to mainland Portugal. Phytocoenologia, 17: 399-415.

Capelo J, Sequeira M, Jardim R, Costa J C, Mesquita, S (2004) Guia da excursão geobotânica dos V Encontros ALFA 2004 à Ilha da Madeira. In Capelo J (ed.) A paisagem vegetal da ilha da Madeira. Quercetea, 6: 5-45.

Costa J C, Aguiar C, Capelo J, Lousã M, Neto C (1998) Biogeografia de Portugal Continental. Quercetea, 0: 5-56.

Costa J C, Espirito-Santo M D, Lousã M, Rodriguez P, Capelo J, Arsénio P (2002) Flora e vegetação do divisório português. Excursão geobotânica ao costeiro português, Olissiponense e Sintrano. Actas do VII Simpósio da Associação Ibero-Macarronésica de Jardins Botânicos: 249-340.

Cross J R (2006) The potential natural vegetation of Ireland. Biology and Environment: Proceedings of the Royal Irish Academy, 106B (2): 65-116.

Cross J R (1998) An outline and map of the potential natural vegetation of Ireland. Applied Vegetation Science, 1: 241-252.

Gaspar N (2003) Comunidades vegetais do Ribatejo. Dissertação de doutoramento, Instituto Superior de Agronomia, Lisboa.

Hastings A, Hom C L, Ellner S, Turchin P, Godfray H C J (1993) Chaos in ecology: is mother nature a strange attractor? Annu. Rev. Ecol. Syst., 24: 1-33. 
Honrado J (2003) Flora vascular e vegetação natural do parque nacional da Peneda-Gerês. Disssertação de doutoramento. Universidade do Porto, Porto.

IDRHa (1994) Carta de solos de Portugal, 1: 25 000. Instituto de Desenvolvimento Rural e Hidráulica.

Kalkhoven J T R, Van der Werf S (1988) Mapping the potential natural vegetation. In Küchler A W, Zonnenveld I S (eds) Handbook of vegetation science 10, vegetation mapping. Kluwer Academic, Dordrecht: 375-386.

Kelly A, Powell D C, Riggs R A (2005) Predicting potential natural vegetation in an interior northwest landscape using classification tree modeling and a GIS. West. J. Appl. For., 20(2): 117-127.

Küchler A W (1964) Potential natural vegetation of the conterminous United States. American Geographical Society Special Publication, 36. New York.

Lawrence P J, Chase T N (2007) Climate impacts of potential vegetation versus current day MODIS Land Cover in the community climate system model (CCSM). In American Geophysical Union, Fall Meeting 2007.

Lopes M C (2001) A flora e vegetação das terras de Sicó. Dissertação de doutoramento, Instituto Superior de Agronomia, Lisboa.

Mesquita S (2005) Modelação bioclimática de Portugal Continental. Dissertação de mestrado, Instituto Superior de Agronomia, Lisboa.

Miller J, Franklin J (2002) Modeling the distribution of four vegetation alliances using generalized linear models and classification trees with spatial dependence. Ecological Modelling, 157: 227-247.

Miller J, Franklin J, Aspinal R (2007) Incorporating spatial dependence in predictive vegetation models. Ecological Modelling, 202: 225-242.

Moravec J (1998) Reconstructed natural versus potential natural vegetation in vegetation mapping - a discussion of concepts. Applied Vegetation Science, 1(2): 173-176.

Neto C (1999) A flora e a vegetação da faixa litoral entre Tróia e Sines. Dissertação de doutoramento, Faculdade de Letras da Universidade de Lisboa, Lisboa.

Neto C, Capelo J, Costa J C (2001) Cartografia fitossociológica da vegetação do Estuário do Sado e Costa da Galé (Portugal). In Mercado G, Poveda F, Mota J F (Eds.) Vegetación y cambios climáticos: 435-446.

Neuhäuslová Z (2001) Potential natural vegetation of the Czech Republic. Braun-Blanquetia, 30: $1-80$.

Neuhäuslová Z (1998) (ed) Map of the potential natural vegetation of the Czech Republic. Academia, Prague.

Pinto Gomes C, Paiva Ferreira R (2005) Flora e vegetação do Barrocal Algarvio. Tavira-Portimão. Comissão de Coordenação e Desenvolvimento do Algarve.

Ricotta C, Carranza M L, Avena G, Blasi C (2002) Are potential natural vegetation maps a meaningful alternative to neutral landscape models? Applied Vegetation Science, 5: 271-275.

Rivas-MartínezS (2005) Notions on dynamic-catenal phytosociology as a basis of landscape science. Plant Biosystems, 139(2): 135-144.

Rivas-Martínez S (1994) Dynamic-zonal phytosociology as landscape science. Phytocoenologia, 24: 23-25.

Rivas-Martínez S (1987) Memória del mapa de séries de vegetación de España 1: 400.000. ICONA, Madrid.

Rivas-Martínez S (1976) Sinfitosociología, una nueva metodologia para el studio del paisage vegetal. Anales Inst. Bot. Cavanilles, 33: 179-188. 
Rivas-Martínez S, Lousã M, Díaz González T E, Fernández-González F, Costa J C (1990) La vegetación del sur de Portugal (Sado, Alentejo y Algarve). Itinera Geobotanica, 3: 5- 126.

Stone L, Ezrati S. (1996) Chaos, cycles and spatiotemporal dynamics in plant ecology. J. Ecol. 84: 279-291.

Theurillat J P (1992a) Étude et Cartographie du Paysage Végétal (Symphytocoenologie) Dans la Région d'Aletsch (Valais, Suisse). Développement Historique et Conceptuel de la Symphytosociologie, Niveaux de Perception, Méthodologie, Applications I. -Texte. - Beiträge zur geobotanischen Landesaufnhame der Schweiz 68. Comission géobotanique de 1 'Académie Suisse des Sciences Naturelles. Krypto F. Flück-Wirth. Lausanne et Genève. Suisse.

Theurillat J P (1992b) L'analyse du paysage végétale en symphytocoenologie: ses niveaux et leurs domaines spatiaux. Bull. Ecol. 23: 83-92.

Trautmann W (1966) Erläuterungen zur karte der potentiellen natuärlichen vegetation der Bundesrepublik Deutschland 1:200 000, Blatt 85 Minden. Schriftenreihe Vegetationskunde, 1: 1137.

Tüxen R (1979) Sigmeten und geosigmeten, irhe ordnung und ihre bedeutung für wissenschaft, naturschutz und planung. Biogeographie, 16: 79-92.

Tüxen R (1978) Assoziationskomplexe (Sigmeten) und Ihre Praktische. Anwendung. Ber. Int. Symp. Int. Vereinigung Vegetationsk.

Tüxen R (1977) Zur homogenität von sigmassociazonen ihrer syntaxonomischen ordnung und ihrer verwendung in der vegetationkartierung. Doc. Phytosociol., N.S., 1: 321-327Tüxen R (1956) Die heutige potentielle natürliche vegetation als gegenstan der vegetationskartierung. Angewandte Pflanzensoziologie, 13: 5-42.

Tüxen R (1973) Vorschlag zur aufnahme von gesellschaftkomplexen in potentiell natürlichen vegetationsgebieten. Acta Bot. Acad. Sci. Hung., 19: 379-384.

Vuerich L G, Poldini L, Feoli E (2001) Model for the potential natural vegetation mapping of friuli venezia-giulia (ne italy) and its application for a biogeographic classification of the region. Plant Biosystems, 135(3): 319-336.

Westhoff V, Van der Maarel E (1973) The braun-blanquet approach. In R H Whittaker (ed.) Handbook of vegetation science, part v: ordination and classification of communities. The Hague, Junk: 617-726.

Zampieri M, Lionello P (2008) A statistical model for potential natural vegetation, application to mediterranean climate. In EGU Geophysical Research Abstracts, Vol. 10, EGU General Assembly 2008.

Zbyszewski G, Moitinho de Almeida F (1960) Carta Geológica de Portugal na escala de 1/50 000. Notícia explicativa da folha 26-D Caldas da Rainha. Serviços Geológicos de Portugal, Lisboa.

Zbyszewski G, Veiga Ferreira O (1966) Carta Geológica de Portugal na escala de 1/50 000. Notícia Explicativa da Folha 30-B Bombarral. Serviços Geológicos de Portugal, Lisboa.

Zhao C, Chen G, Feng Z (2004) GIS assisted modeling of potential vegetation patterns in zhulihe river watershed of the west-central loess plateau. China. 